УДК 539.3(075.8)

\title{
Solution of the Beltrami Equations for the Infinite Hollow Cylinder under the Axisymmetric Deformation
}

\author{
Peter I. Anferov* \\ Institute of Mathematics and Computer Science \\ Siberian Federal University, \\ Svobodny, 79, Krasnoyarsk, 660041 \\ Russia \\ Christina Y. Aturgasheva ${ }^{\dagger}$
}

Academician M.F. Reshetnev Information Satellite Systems Lenin Street, 52, Zheleznogorsk, Krasnoyarsk region, 662972

Russia

Received 30.09.2016, received in revised form 10.11.2016, accepted 20.04.2017

In this paper, axisymmetric stresses of an infinitely long hollow circular cylinder are found from the strain compatibility equations that are reduced to a system of Bessel-type ordinary differential equations by the Fourier transform. The constants of integration of the expressions for the Fourier transforms of stresses are determined by the Fourier-transformed equilibrium equations and boundary conditions. Numerical results are given for the particular case of cylinder's load.

Keywords: infinitely long cylinder, axisymmetric deformation, Beltrami strain compatibility equations, equilibrium equations, Fourier transform.

DOI: 10.17516/1997-1397-2018-11-1-10-17.

In this paper we proposed a solution difficult stresses problem: to calculate the stress state of infinite hollow cylinder under various loads on the lateral surfaces. The urgency of this problem is determined by the fact that the construction of a cylindrical form is widely used in aircraft industry, mineral resource industry, oil and gas industry, heat and power engineering and building industry.

Solutions to many problems encountered in the practice of the stress state of hollow cylinders obtained by stress functions are given in [1]. Stress functions satisfy the certain differential equations, which are obtained from the equilibrium equations of forces, the strain compatibility equations and Hooke's law. More often used Love's biharmonic stresses function for solving axisymmetric problems of the elasticity theory $[2,3]$. With the mentioned function a solution of elasticity theory for an infinite hollow cylinder under the action of an axisymmetric load on the part of the lateral surface was obtained in [4].

In this paper, axisymmetric stress state of infinite hollow cylinder is determined immediately from the solution of the boundary value problem for the system of the Beltrami strain compatibility equations without stress functions.

\footnotetext{
*AnferovPI@mail.ru

†AturgashevaKY@yandex.ru

(C) Siberian Federal University. All rights reserved
} 


\section{Formulation of the problem}

Let the infinite elastic cylinder $(a \leqslant r \leqslant b,|z|<\infty)$ is loaded on the lateral surface part by the surface force having components $F_{r}(z), F_{z}(z)$ that don't depend on the angular coordinate Fig. 1.

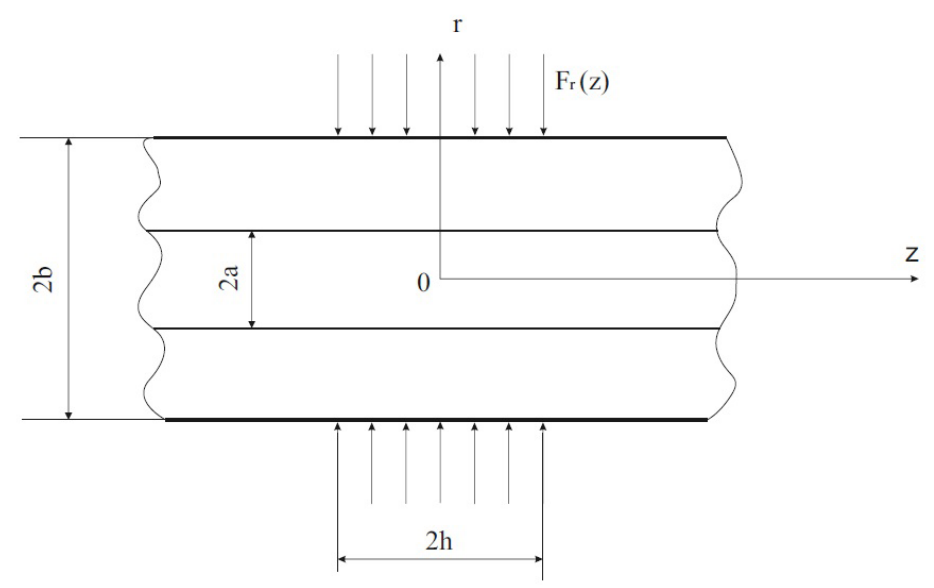

Fig. 1. Scheme of the cylinder loading

The stress tensor components at axisymmetric deformation $\sigma_{j k}, j, k=r, \theta, z$ are described by the Beltrami equations [1]:

$$
\begin{gathered}
\sigma_{r r, r r}+r^{-1} \sigma_{r r, r}+\sigma_{r r, z z}-2 r^{-2}\left(\sigma_{r r}-\sigma_{\theta \theta}\right)=-2 \sigma_{, r r}, \\
\sigma_{\theta \theta, r r}+r^{-1} \sigma_{\theta \theta, r}+\sigma_{\theta \theta, z z}+2 r^{-2}\left(\sigma_{r r}-\sigma_{\theta \theta}\right)=-2 r^{-1} \sigma_{, r}, \\
\sigma_{r z, r r}+r^{-1} \sigma_{r z, r}+\sigma_{r z, z z}-r^{-2} \sigma_{r z}=-2 \sigma_{, r z} ; \\
\sigma_{, r r}+r^{-1} \sigma_{, r}+\sigma_{, z z}=0 .
\end{gathered}
$$

A comma at a subscript indicates the partial differentiation over the coordinates specified after the comma.

The harmonic function $\sigma$ is related to the diagonal components of the stress tensor and the Poisson's ratio $\mu$ by the equation

$$
2(1+\mu) \sigma=\sigma_{r r}+\sigma_{\theta \theta}+\sigma_{z z} .
$$

This function is impossible to find by solving the Dirichlet problem for Laplace's equation by the known methods, because its value can't be set on the boundary of the cylinder.

The system of equations (1)-(4) is the system of eighth order. The order of the original system is artificially increased in its derivation [1]. As a result, possible solutions of the system (1)-(4) generate a class of functions greater than possible solutions of the problem of the elasticity theory [5]. Therefore, we require that the functions $\sigma_{j k}, j, k=r, \theta, z$, that detected from the Beltrami equations, have to identically satisfy the equilibrium equations:

$$
\begin{gathered}
r \sigma_{r r, r r}+\left(\sigma_{r r}-\sigma_{\theta \theta}\right)+r \sigma_{r z, z}=0, \\
r \sigma_{z z, z}+r \sigma_{r z, r}+\sigma_{r z}=0 .
\end{gathered}
$$


Boundary conditions for (1)-(4):

$$
\begin{gathered}
\sigma_{r z}(a, z)=\sigma_{r r}(a, z) \equiv 0, \\
\sigma_{r r}(b, z)=F_{r}(z) \\
\sigma_{r z}(b, z)=F_{z}(z)
\end{gathered}
$$

The stress functions are represented as the Fourier integrals in complex form

$$
\sigma_{j k}(r, z)=\frac{1}{\sqrt{2 \pi}} \int_{-\infty}^{\infty} \bar{\sigma}_{j k}(r, \omega) \exp (i \omega z) d \omega, \quad j, k=r, \theta, z
$$

Here and below overline denotes the exponential Fourier transform of function:

$$
\bar{\sigma}_{j k}(r, \omega)=\frac{1}{\sqrt{2 \pi}} \int_{-\infty}^{\infty} \sigma_{j k}(r, z) \exp (-i \omega z) d z, \quad j, k=r, \theta, z .
$$

\section{The calculation of the Fourier transform of the stresses}

Multiplying all terms of equations (1)-(4) and (6), (7) by $\frac{1}{\sqrt{2 \pi}} \exp (-i \omega z)$ and integrating with respect to $\mathrm{z}$ from $-\infty$ to $+\infty$, we derive the following system of the ODE:

$$
\begin{gathered}
\frac{d^{2} \bar{\sigma}_{r r}}{d r^{2}}+\frac{1}{r} \frac{d \bar{\sigma}_{r r}}{d r}-\omega^{2} \bar{\sigma}_{r r}-\frac{2}{r^{2}}\left(\bar{\sigma}_{r r}-\bar{\sigma}_{\theta \theta}\right)=-2 \frac{d^{2} \bar{\sigma}}{d r^{2}} \\
\frac{d^{2} \bar{\sigma}_{\theta \theta}}{d r^{2}}+\frac{1}{r} \frac{d \bar{\sigma}_{\theta \theta}}{d r}-\omega^{2} \bar{\sigma}_{\theta \theta}+\frac{2}{r^{2}}\left(\bar{\sigma}_{r r}-\bar{\sigma}_{\theta \theta}\right)=-\frac{2}{r} \frac{d \bar{\sigma}}{d r} \\
\frac{d^{2} \bar{\sigma}_{r z}}{d r^{2}}+\frac{1}{r} \frac{d \bar{\sigma}_{r z}}{d r}-\omega^{2} \bar{\sigma}_{r z}-\frac{1}{r^{2}} \bar{\sigma}_{r z}=-2 i \omega \frac{d \bar{\sigma}}{d r}, \\
\frac{d^{2} \bar{\sigma}}{d r^{2}}+\frac{1}{r} \frac{d \bar{\sigma}}{d r}-\omega^{2} \bar{\sigma}=0 \\
r \frac{d \bar{\sigma}_{r r}}{d r}+\left(\bar{\sigma}_{r r}-\bar{\sigma}_{\theta \theta}\right)+i \omega r \bar{\sigma}_{r z}=0 \\
i \omega r \bar{\sigma}_{z z}+r \frac{d \bar{\sigma}_{r z}}{d r}+\sigma_{r z}=0 .
\end{gathered}
$$

We turn to the new independent variable $\rho=\omega r$ in equations $(13)-(16),(17),(18)$ :

$$
\begin{gathered}
\bar{\sigma}_{r r}^{\prime \prime}+\rho^{-1} \bar{\sigma}_{r r}^{\prime}-\bar{\sigma}_{r r}-2 \rho^{-2}\left(\bar{\sigma}_{r r}-\bar{\sigma}_{\theta \theta}\right)=-2 \bar{\sigma}^{\prime \prime}, \\
\bar{\sigma}_{\theta \theta}^{\prime \prime}+\rho^{-1} \bar{\sigma}_{\theta \theta}^{\prime}-\bar{\sigma}_{\theta \theta}+2 \rho^{-2}\left(\bar{\sigma}_{r r}-\bar{\sigma}_{\theta \theta}\right)=-2 \rho^{-1} \bar{\sigma}^{\prime}, \\
\bar{\sigma}_{r z}^{\prime \prime}+\rho^{-1} \bar{\sigma}_{r z}^{\prime}-\left(1+\rho^{-2}\right) \bar{\sigma}_{r z}=-2 i \bar{\sigma}^{\prime}, \\
\bar{\sigma}^{\prime \prime}+\rho^{-1} \bar{\sigma}^{\prime}-\bar{\sigma}=0 \\
\rho \bar{\sigma}_{r r}^{\prime}+\left(\bar{\sigma}_{r r}-\bar{\sigma}_{\theta \theta}\right)+i \rho \bar{\sigma}_{r z}=0 \\
i \rho \bar{\sigma}_{z z}+\rho \bar{\sigma}_{r z}^{\prime}+\bar{\sigma}_{r z}=0 .
\end{gathered}
$$

Hereinafter the primes indicate differentiation on $\rho$. 
The boundary conditions for equations (19)-(22) follow from the Fourier-transformed equations (8)-(10):

$$
\begin{gathered}
\bar{\sigma}_{r z}(\omega a)=\bar{\sigma}_{r r}(\omega a) \equiv 0, \\
\bar{\sigma}_{r r}(\omega b)=\bar{F}_{r}(\omega), \\
\bar{\sigma}_{r z}(\omega b)=\bar{F}_{z}(\omega) .
\end{gathered}
$$

Values of $\bar{\sigma}_{r r}, \bar{\sigma}_{\theta \theta}$ are obtained from the known solution of Lame's problem $[6,7]$ at $\omega=0$. We introduce some new unknown functions in order to integrate equations (19) and (20):

$$
\begin{aligned}
& s_{1}(\rho)=\bar{\sigma}_{r r}(\rho)+\bar{\sigma}_{\theta \theta}(\rho), \\
& s_{2}(\rho)=\bar{\sigma}_{r r}(\rho)-\bar{\sigma}_{\theta \theta}(\rho),
\end{aligned}
$$

from which $\bar{\sigma}_{r r}$ and $\bar{\sigma}_{\theta \theta}$ are expressed by formulas:

$$
\begin{aligned}
& \bar{\sigma}_{r r}=\frac{1}{2}\left[s_{1}(\rho)+s_{2}(\rho)\right], \\
& \bar{\sigma}_{\theta \theta}=\frac{1}{2}\left[s_{1}(\rho)-s_{2}(\rho)\right] .
\end{aligned}
$$

Adding and subtracting equations (19), (20) term by term, and considering expressions (28), (29) and (22), we determine:

$$
\begin{gathered}
s_{1}^{\prime \prime}+\rho^{-1} s_{1}^{\prime}-s_{1}=-2 \bar{\sigma} \\
s_{2}^{\prime \prime}+\rho^{-1} s_{2}^{\prime}-\left(1+4 \rho^{-2}\right) s_{2}=-2\left(\bar{\sigma}-2 \rho^{-1} \bar{\sigma}^{\prime}\right) .
\end{gathered}
$$

The general solution of (22) is

$$
\bar{\sigma}(\rho)=A I_{0}(\rho)+B K_{0}(\rho),
$$

where $A, B$ are any constants, $I_{0}(\rho), K_{0}(\rho)$ are the modified Bessel functions of zero order.

Using the differentiation formulas, the recurrence relations for the Bessel functions [7] and equation (34), we find

$$
\begin{gathered}
\bar{\sigma}^{\prime}(\rho)=A I_{1}(\rho)-B K_{1}(\rho), \\
2 \rho^{-1} \bar{\sigma}^{\prime}(\rho)=-A I_{2}(\rho)-B K_{2}(\rho)+\bar{\sigma}(\rho) .
\end{gathered}
$$

Substituting formulas (34), (36), (35) in the right side of equations (19), (20), (21) we rewrite:

$$
\begin{gathered}
s_{1}^{\prime \prime}+\rho^{-1} s_{1}^{\prime}-s_{1}=-2\left(A I_{0}(\rho)+B K_{0}(\rho)\right), \\
s_{2}^{\prime \prime}+\rho^{-1} s_{2}^{\prime}-\left(1+4 \rho^{-2}\right) s_{2}=-2\left(A I_{2}(\rho)+B K_{2}(\rho)\right), \\
\bar{\sigma}_{r z}^{\prime \prime}+\rho^{-1} \bar{\sigma}_{r z}^{\prime}-\left(1+\rho^{-2}\right) \bar{\sigma}_{r z}=-2 i\left(A I_{1}(\rho)-B K_{1}(\rho)\right) .
\end{gathered}
$$

Equations (37)-(39) are the special cases of the heterogeneous modified Bessel equation of $n$-order

$$
y^{\prime \prime}+\rho^{-1} y^{\prime}-\left(1+n^{2} \rho^{-2}\right) y=2\left(M I_{n}+N K_{n}\right), \quad n=0,1,2 .
$$

Its particular solutions are given by

$$
\begin{aligned}
& y_{1}(\rho)=\rho\left[M I_{n-1}(\rho)-N K_{n-1}(\rho)\right], \\
& y_{2}(\rho)=\rho\left[M I_{n+1}(\rho)-N K_{n+1}(\rho)\right] .
\end{aligned}
$$


Giving values of the corresponding numbers in the right parts of equations (37)-(39) to constants $n, M$ и $N$ of equations (40)-(42), wе can write their general solutions

$$
\begin{aligned}
& s_{1}(\rho)=A_{1} I_{0}(\rho)+B_{1} K_{0}(\rho)+\rho\left[-A I_{1}(\rho)+B K_{1}(\rho)\right], \\
& s_{2}(\rho)=A_{2} I_{2}(\rho)+B_{2} K_{2}(\rho)+\rho\left[-A I_{1}(\rho)+B K_{1}(\rho)\right], \\
& \bar{\sigma}_{r z}(\rho)=A_{3} I_{1}(\rho)+B_{3} K_{1}(\rho)-i \rho\left[A I_{0}(\rho)+B K_{0}(\rho)\right],
\end{aligned}
$$

where $A_{k}, B_{k}, k=1,2,3$ are any constants.

We express $\bar{\sigma}_{z z}$ from the Fourier-transformed formula (5) taking into account (28):

$$
\bar{\sigma}_{z z}=2(1+\mu) \bar{\sigma}(\rho)-s_{1}(\rho) .
$$

Represent $\bar{\sigma}_{r r}, \bar{\sigma}_{\theta \theta}, \bar{\sigma}_{z z}$ in equations (23), (24) by formulas (30), (31), (46) and derive two equations, which functions $\bar{\sigma}, s_{1}, s_{2}, \bar{\sigma}_{r z}$ have to satisfy identically

$$
\begin{gathered}
\rho s_{1}^{\prime}+\rho s_{2}^{\prime}+2 s_{2}+2 i \rho \bar{\sigma}_{r z}=0, \\
i \rho\left[2(1+\mu) \bar{\sigma}-s_{1}\right]+\rho \bar{\sigma}_{r z}^{\prime}+\bar{\sigma}_{r z}=0 .
\end{gathered}
$$

Identities (47), (48) allow establishing communication between constants in formulas (43)-(45).

For the linear combination of the modified Bessel functions

$$
\begin{aligned}
& y_{n}(\rho)=M I_{n}(\rho)+N K_{n}(\rho), \\
& z_{n}(\rho)=\rho\left[R I_{n}(\rho)+S K_{n}(\rho)\right]
\end{aligned}
$$

the following equations [7] are correct

$$
\begin{gathered}
\rho y_{n}^{\prime}(\rho)+n y_{n}(\rho)=\rho\left[M I_{n-1}(\rho)-N K_{n-1}(\rho)\right], \\
\rho z_{n}^{\prime}(\rho)+(n-1) z_{n}(\rho)=\rho^{2}\left[R I_{n-1}(\rho)-S K_{n-1}(\rho)\right] .
\end{gathered}
$$

We substitute $s_{1}(\rho), s_{2}(\rho), \bar{\sigma}_{r z}(\rho), \sigma(\rho)$ in formulas (47), (48) for equations (43), (44), (45) and (34). Using expressions (49)-(52), after transformations, we obtain two identities:

$$
\begin{gathered}
\left(A_{1}+A_{2}-2 A+2 i A_{3}\right) I_{1}(\rho)+\left(-B_{1}-B_{2}+2 B+2 i B_{3}\right) K_{1}(\rho) \equiv 0, \\
\left(2 \mu A-A_{1}-i A_{3}\right) I_{0}(\rho)+\left(2 \mu B-B_{1}+i B_{3}\right) K_{0}(\rho) \equiv 0 .
\end{gathered}
$$

In recording (54) we take into account that $I_{-1}(\rho)=I_{1}(\rho), K_{-1}(\rho)=K_{1}(\rho)$.

The expressions in parentheses in formulas (53), (54) have to equal zero, because $I_{n}(\rho)$, $K_{n}(\rho)$ are the linear independent functions by definition. This requirement will be satisfied if the constants are related by the following equations:

$$
\begin{array}{cl}
A_{2}=A_{1}-2(2 \mu-1) A, & B_{2}=B_{1}-2(2 \mu-1) B, \\
A_{3}=i\left(A_{1}-2 \mu A\right), & B_{3}=i\left(2 \mu B-B_{1}\right) .
\end{array}
$$

We replace $A_{2}, B_{2}, A_{3}, B_{3}$ in equalities (44), (45) by formulas (55), (56), respectively, and, considering expressions $(30),(31)$, we can write for $\bar{\sigma}_{j k}$ :

$$
\begin{gathered}
2 \bar{\sigma}_{r r}=A_{1} C_{1}(\rho)+B_{1} C_{2}(\rho)-A C_{3}(\rho)+B C_{4}(\rho), \\
\bar{\sigma}_{r z}=i\left(A_{1} I_{1}(\rho)-B_{1} K_{1}(\rho)+A C_{5}(\rho)+B C_{6}(\rho)\right), \\
2 \bar{\sigma}_{\theta \theta}=A_{1}\left[I_{0}(\rho)-I_{2}(\rho)\right]+B_{1}\left[K_{0}(\rho)-K_{2}(\rho)\right]+2(2 \mu-1) A I_{2}(\rho)+2(2 \mu-1) B K_{2}(\rho) .
\end{gathered}
$$


Subjecting $\bar{\sigma}_{r r}, \bar{\sigma}_{r z}$ to the boundary conditions (25)-(27), obtain a system of algebraic equations for calculating the constants $A, B, A_{1}, B_{1}$ :

$$
\begin{gathered}
A_{1} C_{1}(b \omega)+B_{1} C_{2}(b \omega)-A C_{3}(b \omega)+B C_{4}(b \omega)=2 \bar{F}_{r}(\omega), \\
A_{1} I_{1}(b \omega)-B_{1} K_{1}(b \omega)+A C_{5}(b \omega)+B C_{6}(b \omega)=-i \bar{F}_{z}(\omega), \\
A_{1} C_{1}(a \omega)+B_{1} C_{2}(a \omega)-A C_{3}(a \omega)+B C_{4}(a \omega)=0 \\
A_{1} I_{1}(a \omega)-B_{1} K_{1}(a \omega)+A C_{5}(a \omega)+B C_{6}(a \omega)=0,
\end{gathered}
$$

where

$$
\begin{gathered}
C_{1}(\rho)=I_{0}(\rho)+I_{2}(\rho), \quad C_{2}(\rho)=K_{0}(\rho)+K_{2}(\rho), \\
C_{3}(\rho)=2 \rho I_{1}(\rho)+2(2 \mu-1) I_{2}(\rho), \quad C_{4}(\rho)=2 \rho K_{1}(\rho)-2(2 \mu-1) K_{2}(\rho), \\
C_{5}(\rho)=-2 \mu I_{1}(\rho)-\rho I_{0}(\rho), \quad C_{6}=2 \mu K_{1}(\rho)-\rho K_{0}(\rho) .
\end{gathered}
$$

Solving the system of equations (60)-(63), we determine the constants in formulas (34), (43), (57)-(59) expressing the Fourier transform of the stresses. The inverse Fourier transforms (11) are carried out numerically.

The coefficients $B, B_{1}, B_{2}, B_{3}$ have to set equal to zero in formulas (34), (43)-(45) for $\bar{\sigma}$, $s_{1}, s_{2}, \bar{\sigma}_{r z}$ and eliminate boundary conditions (8) and (25) to apply the above dependencies for calculating the stresses in a solid cylinder.

\section{An example of the numerical simulation}

In this paper, we calculate the stresses of the hollow cylinder $0,5 b \leqslant r \leqslant b$ which the outer surface is subjected to the radial load $F_{r}(z)$ distributing by the following law:

$$
F_{r}(z)=\left\{\begin{array}{c}
-p_{0} \cos ^{2}\left(\frac{\pi z}{2 h}\right),|z| \leqslant h, p_{0}=\mathrm{const} \\
0,|z|>h .
\end{array}\right.
$$

In this case

$$
\bar{F}_{r}(\omega)=\frac{p_{0} \pi^{2} \sin (\omega h)}{\sqrt{2 \pi}\left(\omega^{2} h^{2}-\pi^{2}\right) \omega} .
$$

The calculation of the values of the modified Bessel functions and solution of linear system (60)-(63) are performed by making use of the math library IMSL that is included in the version FPS.4 of the Fortran. Inverse Fourier transform is numerically carried by quadrature rules based on the interpolation of the third order.

The results of the calculations of the stresses in the cylinder are given on Figs. 2-5, taking $h=b, a=b / 2$ and Poisson's ratio $\mu=0,3$.

Fig. 2 shows that the radial stress $\mathrm{z}$ is compressive for all. Calculations indicate that the angular stress $\sigma_{\theta \theta}$ is compressive at $\frac{z}{h}<1,5$ and tensile at $\frac{z}{h}>1,5$ (Fig. 3). Axial stress $\sigma_{z z}$ changes the sign at the point $\frac{r}{h}=0,8$ (Fig. 4), and besides it is tensile at $0<\frac{z}{h}<0,4$ and compressive at $0,4<\frac{z}{h}<\infty$. The shear stress is less in absolute value than other stress and has extremes, if $\frac{r}{h}=0,75$ for all values of $\frac{z}{h}$ and changes sign at $\frac{z}{h}=1$, as shown in Fig. 5 . 


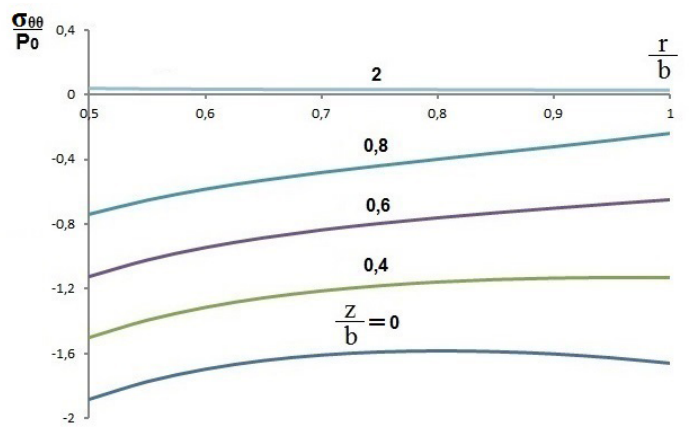

Fig. 2. The distribution of radial stress

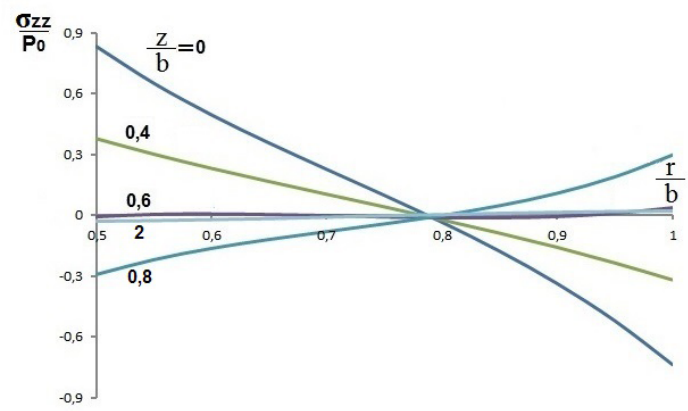

Fig. 4. The distribution of axial stress

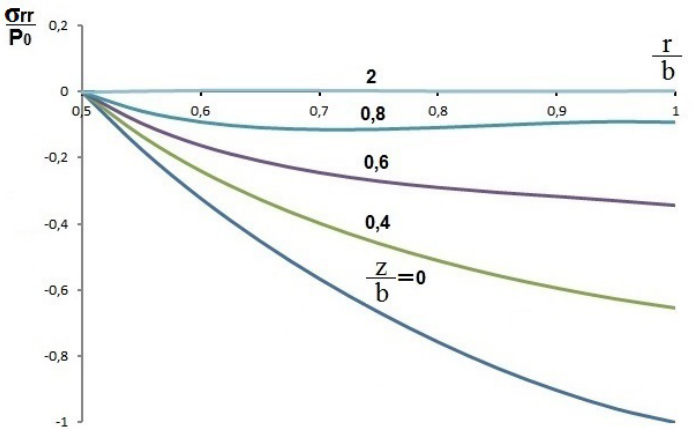

Fig. 3. The distribution of angular stress

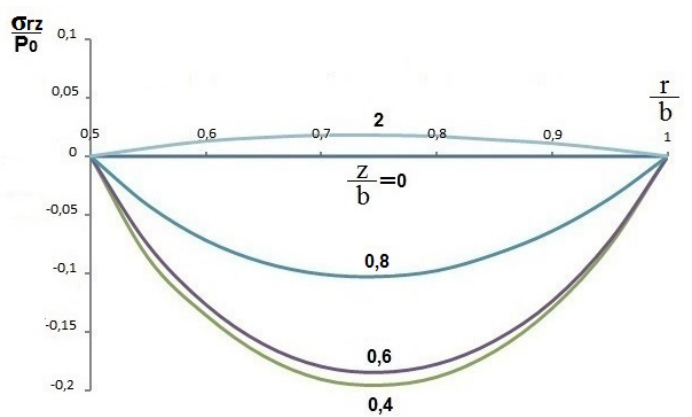

Fig. 5. The distribution of shear stress

\section{References}

[1] M.A.Koltunov, Y.N.Vasilyev, V.A.Chernyh, Elasticity and strength of cylindrical bodies, Moscow, Vysshaya Shkola, 1975 (in Russian).

[2] A.E.Love, Mathematical theory of elasticity, Cambridge, Cambridge Univ., 1927.

[3] A.E.Love, A Treatise on the Mathematical Theory of Elasticity, Cambridge, Cambridge Univ., 1924.

[4] S.Masao, O.Juhachi, Problems on the finite hollow cylinders under the axially symmetrical deformations, Bulletin of JSME, 11(1968), no. 48, 1000-1014.

[5] Y.N.Rabotnov, Mechanics of a deformed solid, Moscow, Nauka, 1979 (in Russian).

[6] G.Lame, Lecons surla theorie de l'elastite, Paris, Gauthier-Villars, 1852.

[7] S.P.Timoshenko, J.N.Goodier, Theory of Elasticity, N.Y., Mc Graw-Hill, 1970. 


\section{Решение уравнений Бельтрами для бесконечного полого цилиндра при осесимметричной деформации}

Пётр И. Анфёров

Институт математики и фундаментальной информатики

Сибирский федеральный университет

Свободный, 79, Красноярск, 660041

Россия

Кристина Ю. Атургашева

АО «Информационные спутниковые системы» имени академика М.Ф. Решетнева»

Ленина, 52, Железногорск Красноярского края, 662972

Россия

В работе найдены осесимметричные напряжения в бесконечном упругом иилиндре из уравнений совместности деформаций, которые преобразованием Фуръе сведены к системе обыкновенных дифференцильных уравнений типа Бесселя. Константы интегрирования, входящие в выражения для трансформант Фуръе напряжений, определяются из преобразованных по Фуръе уравнений равновесия и граничных условий. Для частного случая нагружения иилиндра приведены результаты численных расчетов напряжений.

Ключевые слова: бесконечный иилиндр, осесимметричная деформация, уравнения совместности деформачий Бельтрами, уравнения равновесия, преобразование Фуръе. 